\title{
Controlling Rate and Rhythm Increases Feasiblity of CT Angiography in Atrial Fibrillation
}

\author{
A. Al Fagih, MD; S.A. Al Ghamdi, MD; K. Dagriri, MD; and G. Al Zahrani, MD
}

\begin{abstract}
A woman, aged 48 years, with severe rheumatic mitral stenosis and uncontrolled permanent atrial fibrillation (AF) underwent preoperative assessment of coronary arteries. Invasive coronary angiography was not possible because of occluded common iliac artery and bilateral radial spasm. Transesophageal echocardiogram showed a very large mobile left atrial appendage clot, precluding cardioversion. The severe motion artifacts during cardiac CT angiography (64 slices) due to atrial fibrillation were overcome by controlling rhythm and rate through insertion of a temporary pacemaker via right femoral vein, and slowing heart rate below 65 beats per minute by intravenous metoprolol $(25 \mathrm{mg})$ and verapamil $(5 \mathrm{mg})$. Clear pictures of all coronary arteries as well as the left atrial appendage clot were obtained. The temporary pacemaker was removed after eight hours. Uneventful mechanical mitral valve replacement and maze procedure were performed and the patient was discharged in a stable condition.
\end{abstract}

Keywords: Atrial fibrillation, CT angiography, Rheumatic patient

Reprint Requests:

A. Al Fagih, MD

Department of Adult Cardiology

Prince Sultan Cardiac Center

Riyadh

Kingdom of Saudi Arabia

Tel: $+966|47777| 4$ Ex. 8765

Fax: $+966|477877|$

Email: aafagih@yahoo.com

Received: July II, 2009

Revised: October 10, 2009

Accepted: October 28, 2009

doi: $10.3121 / \mathrm{cmr} .2010 .874$
$\mathrm{R}$ allow for accurate, non-invasive visualization and characterization of atherosclerotic coronary disease and other cardiac abnormalities. ${ }^{1}$ Uncontrolled atrial fibrillation (AF) with significant beat-beat variation may result in motion artifacts that reduce the diagnostic accuracy of $\mathrm{CCT}^{2}$ Therefore, in order to achieve motion-free images with optimal visualization of coronary arteries, computed tomography (CT) data needs to be acquired using scanners with the highest temporal resolution. ${ }^{3}$ The present technique demonstrates that temporary pacing with an aggressive rate control of atrial fibrillation may overcome this limitation.

\section{Case Presentation}

A woman, aged 48 years, with a long-standing history of rheumatic heart disease presented with symptomatic severe mitral valve stenosis and permanent AF with an average ventricular rate of 100 beats per minutes (bpm). She had a strong family history of premature coronary artery disease and typical chest pain requiring pre-operative assessment of coronary arteries. CCT angiography could not be performed initially due to uncontrolled AF despite adequate oral medical therapy. Alternatively, she underwent invasive coronary angiography, wherein bilateral common iliac artery occlusion was found. She also developed bilateral radial spasm during radial approach (figure 1). An elective cardioversion to obtain adequate CCT images during sinus rhythm was precluded by the presence of a large mobile left atrial appendage (LAA) thrombus detected by transesophageal echocardiography (figure 2). Therefore, to overcome the motion artifacts during CCT angiography due to an irregular rhythm, a temporary pacemaker was inserted via the right femoral vein, and then a ventricular rate below $65 \mathrm{bpm}$ was 


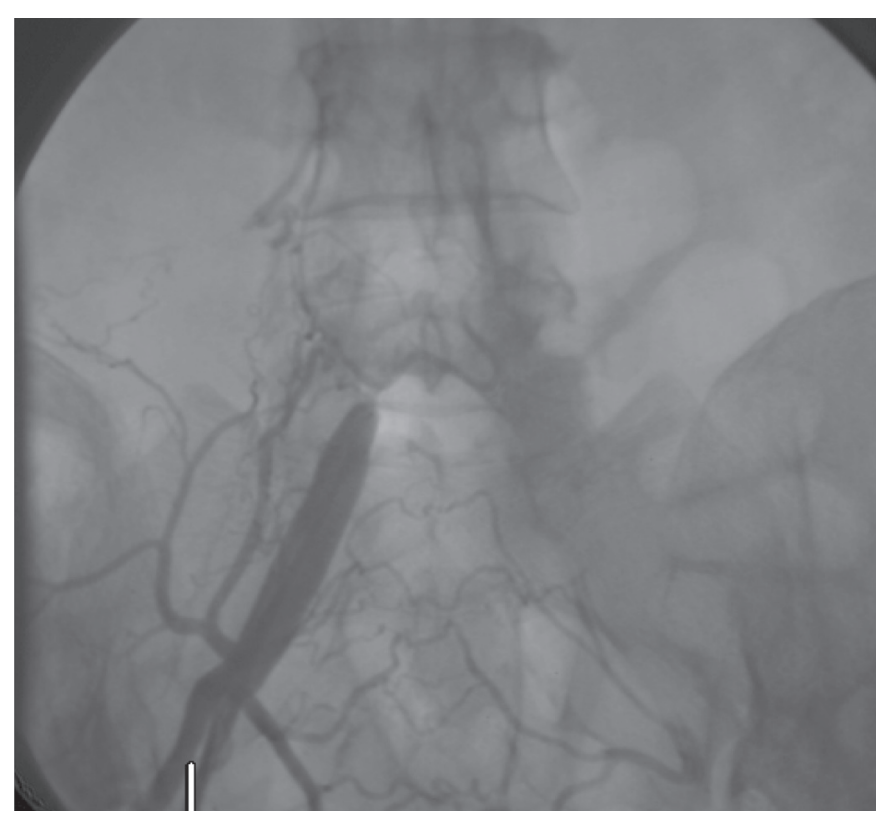

Figure 1. Right femoral artery angiography showed occluded common iliac artery.

achieved by intravenous metoprolol $(25 \mathrm{mg}$ ) and verapamil (5 $\mathrm{mg}$ ). CCT angiography was performed using a single source scanner (Philips Brilliance CT 64 detectors) with retrospective electrocardiogram gating. Images were reconstructed from diastolic data set at $75 \%$ of the R-R interval. Maximum intensity project (MIP), multiplanar reformation (MPR) and 3-dimensional virtual reality images were used to assess the coronary arteries, peripheral vessels and cardiac chambers (figure 3). The patient remained hemodynamically stable throughout and after the procedure. The temporary pacemaker wire was removed 8 hours later with no complications. She underwent an uneventful mechanical mitral valve replacement and was discharged to home in good condition.

\section{Discussion}

With the advent of 64-slice CT, the diagnostic accuracy of CT coronary angiography has improved significantly and fares better in comparison to other currently available methods of

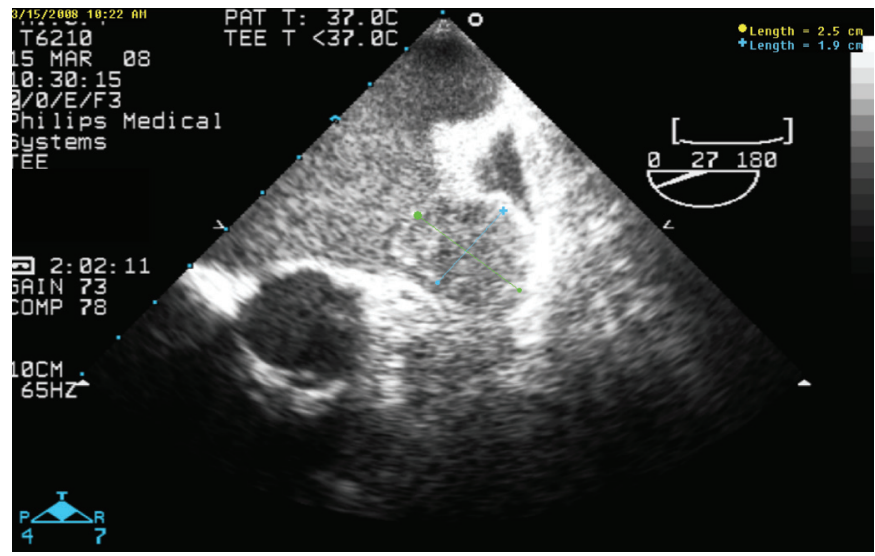

non-invasive coronary imaging, namely echocardiography and cardiac magnetic resonance techniques. ${ }^{1}$ Atrial fibrillation is still considered to be a relative contraindication to CCT despite constant improvements in scanner technology. ${ }^{4-7}$ Inappropriate data sampling occurs due to high beat-beat variations with severe motion artifacts. ${ }^{8,9}$ The latest generation of scanners, including dual source and 256 and 320 detector row systems, have high temporal resolution and are capable of imaging the entire heart in a single gantry rotation, thus reducing the effect of tachyarrhythmias on image quality. 10,11 However, these technologies are not readily available in every center, and therefore, reliable visualization of the coronary arteries remains a major challenge in patients with $\mathrm{AF}$, especially with high beat-beat variations.

The use of a temporary pacemaker to treat a bradyarrhythmia or, in some cases a tachyarrhythmia, is undertaken when the condition is temporary and a permanent pacemaker is not necessary or not available in a timely fashion. ${ }^{12}$ Temporary pacemakers are also used in overdrive atrial pacing to suppress tachyarrhythmias such as atrial flutter, or in overdrive ventricular pacing to treat recurrent ventricular tachycardia or to prevent severe ventricular arrhythmia secondary to prolonged QT interval. ${ }^{12}$

In the present case, conventional coronary angiography failed due to peripheral vasculopathy, while CCT was not an ideal option in the presence of a rapid ventricular response despite medical therapy and the inability of cardioversion to sinus rhythm due to LAA thrombus. In addition, the available CCT was a single source 64-slice detector with a rotation time of $0.42 \mathrm{sec}$. The rapid ventricular rate with high variability mandates aggressive intravenous therapy, which in the present case, was achieved by intravenous metoprolol $(25 \mathrm{mg})$ and verapamil $(5 \mathrm{mg})$. A temporary pacemaker was used to maintain a stable and regular rhythm during scanning to ensure good image quality for interpretation.

In conclusion, by slowing the heart rate using intravenous medication and regulating the rhythm through back up temporary pacing, it is possible to scan patients with AF by CCT angiography.

\section{References}

1. Wilson GT, Gopalakrishnan P, Tak T. Noninvasive cardiac imaging with computed tomography. Clin Med Res 2007;5:165-171.

2. Hoffmann U, Ferencik M, Cury RC, Pena AJ. Coronary CT angiography. J Nucl Med 2006;47:797-806.

3. Nissen SE. Limitations of computed tomography coronary angiography. J Am Coll Cardiol 2008;52:2145-2147.

4. Achenbach S, Giesler T, Ropers D, Ulzheimer S, Derlien H, Schulte C, Wenkel E, Moshage W, Bautz W, Daniel WG, Kalender WA, Baum U. Detection of coronary artery stenoses by contrast-enhanced, retrospectively electrocardiographically-gated, multislice spiral computed tomography. Circulation 2001;103:2535-2538.

Figure 2. Transoesophageal echo showed large left atrial appendage clot. 

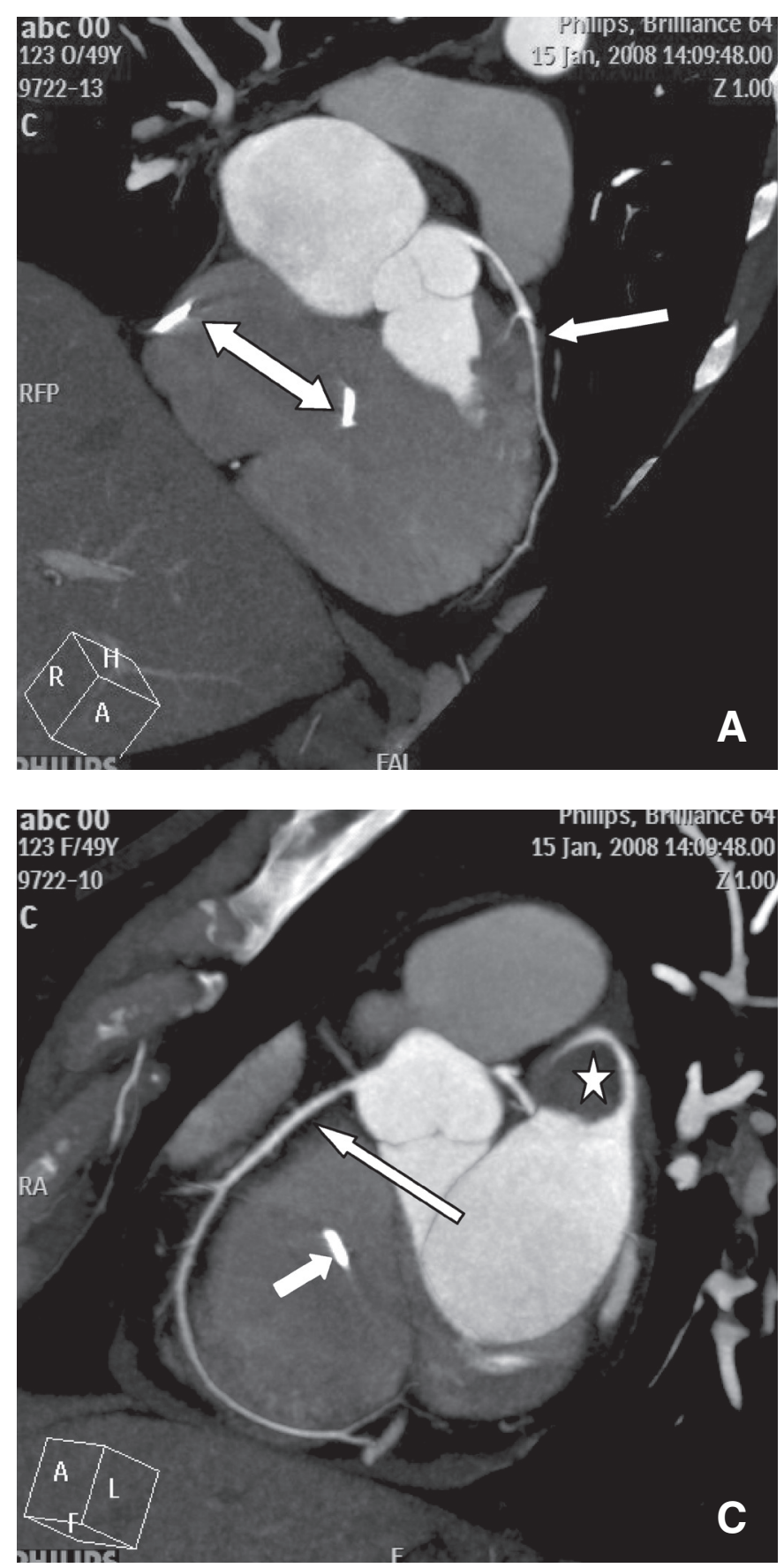

5. Nieman K, Cademartiri F, Lemos PA, Raaijmakers R, Pattynama PM, de Feyter PJ. Reliable noninvasive coronary angiography with fast submillimeter multislice spiral computed tomography. Circulation 2002;106:2051-2054.

6. Heuschmid M, Kuettner A, Schroeder S, Trabold T, Feyer A, Seemann MD, Kuzo R, Claussen CD, Kopp AF. ECG-gated 16-MDCT of the coronary arteries: assessment of image quality and accuracy in detecting stenoses. AJR Am J Roentgenol 2005;184:1413-1419.

7. Pugliese F, Mollet NR, Runza G, van Mieghem C, Meijboom WB, Malagutti P, Baks T, Krestin GP, deFeyter PJ, Cademartiri F. Diagnostic accuracy of non-invasive 64-slice CT coronary angiography in patients with stable angina pectoris. Eur Radiol 2006;16:575-582.

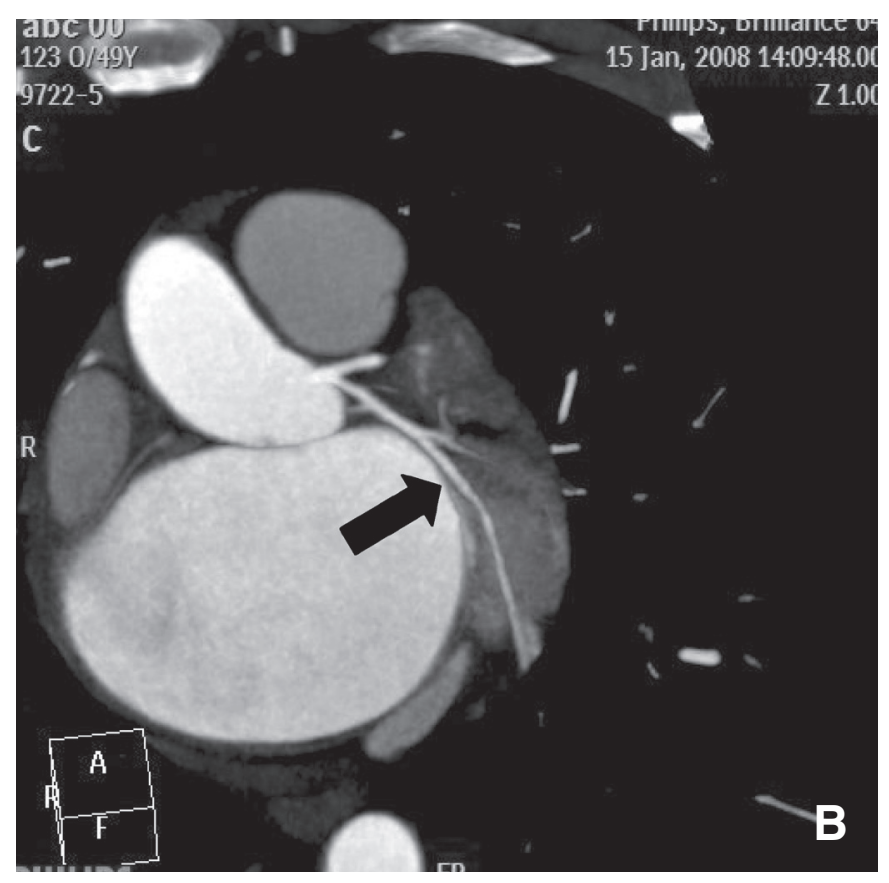

Figure 3. (A) Cardiac computed tomography (CT) with maximum intensity projection view of the left anterior descending artery (single-point white arrow), and part of the temporary pacemaker in right ventricle (double-point white arrow). (B) Cardiac CT with maximum intensity projection view of the left circumflex artery (black arrow). (C) Cardiac CT with maximum intensity projection shows right coronary artery (long arrow), left atrial appendage clot (star) and part of the temporary pacemaker in right ventricle (short arrow).

8. Raff GL, Gallagher MJ, O’Neill WW, Goldstein JA. Diagnostic accuracy of noninvasive coronary angiography using 64-slice spiral computed tomography. J Am Coll Cardiol 2005;46:552-557.

9. Sun Z, Jiang W. Diagnostic value of multislice computed tomography angiography in coronary artery disease: a meta-analysis. Eur J Radiol 2006;60:279-286.

10. Rist C, Johnson TR, Müller-Starck J, Arnoldi E, Saam T, Becker A, Leber AW, Wintersperger BJ, Becker CR, Reiser MF, Nikolaou K. Non-invasive Coronary Angiography Using Dual-Source Computed Tomography in Patients with Atrial Fibrillation. Invest Radiol 2009 Jan 15. [Epub ahead of print].

11. Wang Y, Zhang Z, Kong L, Song L, Merges RD, Chen J, Jin Z. Dual-source CT coronary angiography in patients with atrial fibrillation: comparison with single-source CT. Eur J Radiol. 2008;68:434-441.

12. Timothy PR, Rodeman BJ. Temporary pacemakers in critically ill patients: assessment and management strategies. AACN Clin Issues 2004;15:305-325.

\section{Author Affiliations}

A. Al Fagih, MD*; S.A. Al Ghamdi, MD*;

K. Dagriri, MD*; G. Al Zahrani, $M D^{*}$

* Prince Sultan Cardiac Center, Department of Adult Cardiology, Riyadh, Kingdom of Saudi Arabia 